\title{
THE GAME-THEORETIC VALUE AND THE SPECTRAL RADIUS OF A NONNEGATIVE MATRIX
}

\author{
JOEL E. COHEN ${ }^{1}$ AND SHMUEL FRIEDLAND ${ }^{2}$
}

\begin{abstract}
We relate some minimax functions of matrices to some spectral functions of matrices. If $A$ is a nonnegative $n \times n$ matrix, $v(A)$ is the gametheoretic value of $A$, and $\rho(A)$ is the spectral radius of $A$, then $v(A) \leq \rho(A)$. Necessary and sufficient conditions for $v(A)=\rho(A)$ are given. It follows that if $A$ is nonnegative and irreducible and $n>1$, then $v(A)<\rho(A)$. Also, if, for a real matrix $A$ and a positive matrix $B, v(A, B)=\sup _{X} \inf _{Y} X^{\mathrm{T}} A Y / X^{\mathrm{T}} B Y$ over probability vectors $X$ and $Y$, then for nonnegative, nonsingular $A$ and positive $B, \rho(A B)=\left[v\left(A^{-1}, B\right)\right]^{-1}$.
\end{abstract}

The purpose of this paper is to establish some connections between von Neumann's minimax functions of matrices and the spectral radius of nonnegative matrices. Other connections between game-theoretic and linear-algebraic aspects of matrices have been established by Blackwell (1961) and Raghavan (1978).

Let

$$
P_{n}=\left\{X \in \mathbf{R}^{n} \mid X_{i} \geq 0, \sum X_{i}=1\right\}, \quad P_{n}^{+}=\left\{X \in \mathbf{R}^{n} \mid X_{i}>0, \sum X_{i}=1\right\} .
$$

Where no ambiguity can arise, the subscript $n$ will be dropped.

In game theory the minimax theorem (von Neumann, 1928 [1959]) asserts that for any $m \times n$ real matrix $A$,

$$
\sup _{X \in P_{m}} \inf _{Y \in P_{n}} X^{\mathrm{T}} A Y=\inf _{Y \in P_{n}} \sup _{X \in P_{m}} X^{\mathrm{T}} A Y,
$$

where ${ }^{\mathrm{T}}$ denotes transpose. The common value of both sides of $(1)$ is $v(A)$, the value of $A$.

The spectral radius $\rho(A)$ of an $n \times n$ real matrix $A$ is the maximum of moduli of the eigenvalues of $A$. Following the terminology of Birkhoff and Varga (1958), who also review much of the background assumed here, the spectral prenorm $R(A)$ of $A$ is the maximum of the real parts of the eigenvalues of $A$.

A matrix $A$ is nonnegative, and we write $A \geq 0$, if every element $a_{i j}$ of $A$ is greater than or equal to 0 . A matrix $A$ is positive, and we write $A>0$, if every element is greater than 0 . A matrix $A$ is essentially nonnegative if every element not on the main diagonal is nonnegative. An $n \times n$ matrix $A$ is irreducible if for every $i$ and $j$ there exists a finite sequence of indices $k(0)=i, \ldots, k(r)=j$ such that, for $h=1, \ldots, r, a_{k(h-1), k(h)} \neq 0$.

Received by the editors November 30, 1983 and, in revised form, February 23, 1984.

1980 Mathematics Subject Classification. Primary 15A42, 15A48; Secondary 90D05.

Key words and phrases. Eigenvalue inequality, Perron-Frobenius root, minimax, inverse nonnegative matrix, essentially nonnegative matrix, zero-sum two-person game, Jentzsch's theorem.

${ }^{1}$ Supported in part by U.S. National Science Foundation grant DEB80-11026.

${ }^{2}$ Supported in part by U.S.-Israel Binational Science Foundation grant 2242/80. 
If $A$ is any real $m \times n$ matrix and $J$ is the $m \times n$ matrix in which each element equals 1 , then for any real $c, v(A+c J)=v(A)+c$. So if a sufficiently large constant is added to all elements of $A$ to make $A$ essentially nonnegative, or nonnegative, or positive, according to need, then the same constant is added to the value of $A$.

THEOREM 1. If $A$ is any real $n \times n$ matrix such that, for some $V$ in $P, A V=\lambda V$ where $\lambda \geq 0$, then $v(A) \leq \lambda$.

PROOF. It is well known that if $W$ is a vector of $n$ real numbers, then

$$
\sup _{X \in P} X^{\mathrm{T}} W=\max _{i} W_{i}
$$

and the same holds with $P$ replaced by $P^{+}$. Then with $W=A V$,

$$
\sup _{X \in P} X^{\mathrm{T}} A V=\max _{1 \leq i \leq n}(A V)_{i}=\lambda \max _{1 \leq i \leq n} V_{i} \leq \lambda .
$$

Then

$$
v(A)=\inf _{Y \in P} \sup _{X \in P} X^{\mathrm{T}} A Y \leq \sup _{X \in P} X^{\mathrm{T}} A V \leq \lambda .
$$

COROLLARY 1. If $A$ is an essentially nonnegative $n \times n$ matrix such that $R(A) \geq 0$, then $v(A) \leq R(A)$.

ProOf. Since $A$ is essentially nonnegative, there exists a vector $V$ in $P$ such that $A V=R(A) V$ (e.g., Birkhoff and Varga, 1958, p. 356).

COROLlaRY 2. For any nonnegative $n \times n$ matrix $A, 0 \leq v(A) \leq \rho(A)$.

The upper bound on $v(A)$ given by Theorem 1 can be strictly lower than those given by Corollaries 1 and 2. For example, if $A=\left[\begin{array}{ll}1 & 0 \\ 0 & 2\end{array}\right]$, Theorem 1 gives $v(A) \leq 1$ but $R(A)=\rho(A)=2$. (In fact, $v(A)=2 / 3$.)

COROLlaRY 3. For any real $n \times n$ matrix $A$ and any constant $c$ such that $A+c J \geq 0, v(A) \leq \rho(A+c J)-c$.

THEOREM 2. For any nonnegative $n \times n$ matrix $A$, a necessary and sufficient condition for $v(A)=\rho(A)$ is that there exists an $n \times n$ permutation matrix $S$ such that

$$
\begin{gathered}
S A S^{\mathrm{T}}=B=\left(b_{i j}\right), \quad b_{11}=\rho(A), \quad b_{i 1}=0, \quad i=2, \ldots, n, \\
b_{1 j} \geq \rho(A), \quad j=2, \ldots, n .
\end{gathered}
$$

In particular, if $n \geq 2$ and $A$ is irreducible, then $v(A)<\rho(A)$.

Proof. We consider two cases: $\rho(A)=0$ and $\rho(A)>0$.

First, if $\rho(A)=0$, then by applying the same permutation $S$ to the rows and columns of $A$, we can obtain $B=S A S^{\mathrm{T}}$ in strictly upper triangular form (Gantmacher, 1960, Volume 2, pp. 74-76). Therefore $b_{11}=\rho(A)=0, b_{i 1}=0, i=$ $2, \ldots, n$, and $b_{1 j} \geq \rho(A)=0, j=2, \ldots, n$. Conversely, if $B$ is strictly upper triangular, then $\rho(B)=\rho(A)=0$. But $A \geq 0$ implies $v(A) \geq 0$, and since $\rho(A) \geq v(A)$, we must have $v(A)=\rho(A)=0$.

Second, if $\rho(A)>0$, then $v(A)=\rho(A)$ implies (by (2)) that $\max _{1 \leq i \leq n} V_{i}=1$. The permutation matrix $S$ can be chosen so that $V_{1}=1$. In this case, $V_{2}=$ $\cdots=V_{n}=0$. Because the first column of $B$ is given by $B V=\rho(B) V$ and 
$\rho(B)=\rho(A)$, we have $b_{11}=\rho(A)$ and $b_{i 1}=0, i=2, \ldots, n$. It remains to show that $b_{1 j} \geq \rho(A), j=2, \ldots, n$. But since $v(A)=\min _{Y \in P} Y^{\mathrm{T}} A^{\mathrm{T}} \xi$ for some $\xi$ in $P$, we must have, for $i=1, \ldots, n,\left(A^{\mathrm{T}} \xi\right)_{i} \geq v(A)=\rho(A)$. Then writing $\xi=S \alpha$ and premultiplying by $S^{\mathrm{T}}$, this inequality becomes, for $i=1, \ldots, n,\left(S^{\mathrm{T}} A^{\mathrm{T}} S \alpha\right)_{i}=$ $\left(B^{\mathrm{T}} \alpha\right)_{i} \geq \rho(A)$, where $\alpha$ is in $P$. In particular, $\rho(A) \alpha_{1} \geq \rho(A)$, which implies $\alpha_{1} \geq 1$, i.e., $\alpha_{1}=1$ and $\alpha_{2}=\cdots=\alpha_{n}=0$. Then $\left(B^{\mathrm{T}} \alpha\right)_{i} \geq \rho(A)$, for $i=1, \ldots, n$, implies $b_{1 j} \geq \rho(A)$ for $j=2, \ldots, n$. This establishes (3). Conversely, given (3), we have, by (1),

$$
v(A)=\sup _{X \in P} \inf _{Y \in P} Y^{\mathrm{T}} B^{\mathrm{T}} X \geq \inf _{Y \in P} Y^{\mathrm{T}} B^{\mathrm{T}} \alpha \geq \rho(A) .
$$

Since we have already established that $v(A) \leq \rho(A)$, we must have $v(A)=\rho(A)$.

Theorem 1 can be substantially generalized. Let $F$ and $G$ be cumulative distribution functions such that $F(0)=G(0)=0, F(1)=G(1)=1$. Let $A(x, y)$ be a real-valued function of $(x, y) \in[0,1] \times[0,1]$. Let $A$ be continuous in $x$ and $y$. Under these conditions (e.g. Dresher, 1961 [1981], p. 108), there exist distribution functions $F^{*}, G^{*}$ on $[0,1]$ such that

$$
\sup _{F} \int_{0}^{1} \int_{0}^{1} A(x, y) d F(x) d G^{*}(y)=\inf _{G} \int_{0}^{1} \int_{0}^{1} A(x, y) d F^{*}(x) d G(y) .
$$

Call the common value of both sides $v(A)$, the value of the infinite game specified by the payoff function $A$.

THEOREM 3. Suppose there exists a real-valued function $f$ on $[0,1]$ such that $f \geq 0, \int_{0}^{1} f(x) d x=1$ and, for some $\lambda \geq 0$,

$$
\lambda f(x)=\int_{0}^{1} A(x, y) f(y) d y, \quad x \in[0,1] .
$$

Then $v(A) \leq \lambda$.

Proof. Define the distribution function $V(x)$ on $[0,1]$ by $V(x)=\int_{0}^{x} f(x) d x$. Then

$$
\begin{aligned}
v(A) & =\inf _{G} \int_{0}^{1} \int_{0}^{1} A(x, y) d F^{*}(x) d G(y) \\
& \leq \int_{0}^{1} \int_{0}^{1} A(x, y) d F^{*}(x) d V(y) \\
& =\int_{0}^{1} d F^{*}(x) \int_{0}^{1} A(x, y) f(y) d y \\
& =\int_{0}^{1} d F^{*}(x) \lambda f(x) \leq \lambda .
\end{aligned}
$$

One sufficient condition for there to exist a function $f$ satisfying the hypotheses of Theorem 3 is (Birkhoff, 1957, p. 223) that $A$ be uniformly positive in the sense that $0<\inf _{x, y} A(x, y) \leq \sup _{x, y} A(x, y)<\infty$. In this case $f$ is also continuous and $\lambda>0$.

The inequality $v(A) \leq \rho(A)$ may be far from sharp when more is known about $A$ than is assumed in the hypotheses of Theorem 1 . For example, if $A$ is doubly stochastic (meaning that $A \geq 0$ and the sum of every row of $A$ is 1 and the sum of every column of $A$ is 1 ), then $v(A)=1 / n$ while $\rho(A)=1$. 
It follows from Theorem 2 that if $A>0$ and $n \geq 2$, then $v(A)<\rho(A)$. An open question is how far this inequality may be improved when $A$ is also symmetric.

In 1937 von Neumann generalized his minimax theorem (1) as follows. For any $m \times n$ real matrix $A$ and any $m \times n$ matrix $B>0$, there is a number $v(A, B)$ given by

$$
v(A, B)=\sup _{X \in P_{m}} \inf _{Y \in P_{n}} \frac{X^{\mathrm{T}} A Y}{X^{\mathrm{T}} B Y}=\inf _{Y \in P_{n}} \sup _{X \in P_{m}} \frac{X^{\mathrm{T}} A Y}{X^{\mathrm{T}} B Y} .
$$

The special case of (4) when $B=J$ reduces to (1), i.e., $v(A, J)=v(A)$. Shapley (1953, p. 1099) interprets (4) in terms of a stochastic game when each element of $B$ is a positive number not exceeding 1 . Von Neumann proved (4) by showing that there exist two vectors $X \in P_{m}, Y \in P_{n}$ and a number $\lambda$ such that

$$
\begin{gathered}
\lambda(B Y)_{i} \geq(A Y)_{i}, \quad i=1, \ldots, m, \\
\lambda\left(B^{\mathrm{T}} X\right)_{j} \leq\left(A^{\mathrm{T}} X\right)_{j}, \quad j=1, \ldots, n .
\end{gathered}
$$

(5) implies (4) because (5a) implies

$$
\lambda \geq \max _{i} \frac{(A Y)_{i}}{(B Y)_{i}}=\sup _{U \in P_{m}} \frac{U^{\mathrm{T}} A Y}{U^{\mathrm{T}} B Y} \geq \inf _{V \in P_{n}} \sup _{U \in P_{m}} \frac{U^{\mathrm{T}} A V}{U^{\mathrm{T}} B V},
$$

while (5b) implies

$$
\lambda \leq \min _{j} \frac{\left(A^{\mathrm{T}} X\right)_{j}}{\left(B^{\mathrm{T}} X\right)_{j}}=\inf _{V \in P_{n}} \frac{X^{\mathrm{T}} A V}{X^{\mathrm{T}} B V} \leq \sup _{U \in P_{m}} \inf _{V \in P_{n}} \frac{U^{\mathrm{T}} A V}{U^{\mathrm{T}} B V},
$$

i.e., jointly

$$
\inf _{V \in P_{n}} \sup _{U \in P_{m}} \frac{U^{\mathrm{T}} A V}{U^{\mathrm{T}} B V} \leq \lambda \leq \sup _{U \in P_{m}} \inf _{V \in P_{n}} \frac{U^{\mathrm{T}} A V}{U^{\mathrm{T}} B V} .
$$

However, the reverse inequality

$$
\sup _{U \in P_{m}} \inf _{V \in P_{n}} \frac{U^{\mathrm{T}} A V}{U^{\mathrm{T}} B V} \leq \inf _{V \in P_{n}} \sup _{U \in P_{m}} \frac{U^{\mathrm{T}} A V}{U^{\mathrm{T}} B V}
$$

always holds, so (4) follows from (5), with $v(A, B)=\lambda$ in (6). Loomis (1946) gives an elementary proof of (5).

THEOREM 4. Let $A$ and $B$ be $n \times n$ real matrices with $B>0$. (a) If there is a vector $U \in P$ and a real number $\lambda$ such that $A U=\lambda B U$, then $v(A, B) \leq \lambda$. (b) If there is a vector $V \in P$ and a real number $\mu$ such that $V^{\mathrm{T}} A=\mu V^{\mathrm{T}} B$, then $\mu \leq v(A, B)$.

Proof. Fix $X \in P$. Then

$$
\inf _{Y \in P} \frac{X^{\mathrm{T}} A Y}{X^{\mathrm{T}} B Y} \leq \frac{X^{\mathrm{T}} A U}{X^{\mathrm{T}} B U}=\frac{X^{\mathrm{T}} \lambda B U}{X^{\mathrm{T}} B U}=\lambda .
$$

Since the upper bound $\lambda$ is independent of $X$, we have $v(A, B) \leq \lambda$. Similarly, fix $Y \in P$. Then

$$
\sup _{X \in P} \frac{X^{\mathrm{T}} A Y}{X^{\mathrm{T}} B Y} \geq \frac{V^{\mathrm{T}} A Y}{V^{\mathrm{T}} B Y}=\mu,
$$

so $v(A, B) \geq \mu$. 
THEOREM 5. Let $A$ and $B$ be $n \times n$ real matrices with $B>0$. (a) If $B^{-1} A \geq 0$ (providing $B^{-1}$ exists) or $A^{-1} B \geq 0$, then the assumptions of Theorem 4(a) hold, and $v(A, B) \leq \rho\left(B^{-1} A\right)$ or $v(A, B) \leq\left[\rho\left(A^{-1} B\right)\right]^{-1}$, respectively. (b) If $A B^{-1} \geq 0$ (again providing $B^{-1}$ exists) or $B A^{-1} \geq 0$, then the assumptions of Theorem 4(b) hold, and $\rho\left(A B^{-1}\right) \leq v(A, B)$ or $\left[\rho\left(B A^{-1}\right)\right]^{-1} \leq v(A, B)$, respectively.

PROOF. A nonnegative $n \times n$ matrix has a nonzero nonnegative eigenvector corresponding to its spectral radius (Gantmacher, 1960). (a) If $B^{-1} A \geq 0$, take $\rho\left(B^{-1} A\right)=\lambda$ in Theorem 4(a). If $A^{-1} B \geq 0$, then $\rho\left(A^{-1} B\right)>0$. For otherwise there exists $U \in P$ such that $A^{-1} B U=0$, which implies, since $A$ is nonsingular, that $B U=0$. But this is impossible because $B>0$ and $U \neq 0$. Having established that $\rho\left(A^{-1} B\right)>0$, take $\lambda=1 / \rho\left(A^{-1} B\right)$ in Theorem $4(\mathrm{a})$. The proof of part (b) is similar.

CoRollary 4. Let $A$ and $B$ be $n \times n$ matrices. Let $A$ be nonnegative and nonsingular and let $B$ be positive. Then $\rho(A B)=\rho(B A)=\left[v\left(A^{-1}, B\right)\right]^{-1}$.

Proof. Since $A \geq 0, \operatorname{det}(A) \neq 0$ and $B>0$, both $A B>0$ and $B A>0$. Therefore $\rho(A B)=\rho(B A)>0$. By Theorem $5(\mathrm{a}), v\left(A^{-1}, B\right) \leq[\rho(A B)]^{-1}$. By Theorem 5(b), $[\rho(B A)]^{-1} \leq v\left(A^{-1}, B\right)$.

COROLLARY 5. Let $A^{-1}$ be a nonsingular $n \times n$ matrix with nonnegative inverse $A$, and let $S(A)$ be the sum of the elements of $A$. Then $v\left(A^{-1}\right)=1 / S(A)$.

Proof. Taking $B=J$ in Corollary 4 and reviewing (1) and (4), we find $v\left(A^{-1}, J\right)=v\left(A^{-1}\right)=1 / \rho(A J)=1 / S(A)$, since every column sum of $A J$ equals $S(A)$.

Our results enable numerical procedures for calculating the spectral radius to be used to find the value of certain games and, conversely, enable procedures for solving games (Dresher, 1961 [1981], Chapter 5) to be used to find the spectral radius of certain matrices.

We close with three historical remarks.

First, as a special case of the corollary just proved, take $A=I$. By the Perron theorem, there exist vectors $X, Y \in P^{+}$such that $\lambda B Y=I Y, \lambda X^{\mathrm{T}} B=Y^{\mathrm{T}} I$, where $\lambda=[\rho(B)]^{-1}$. So (5) holds. Then von Neumann's theorem (4) yields

$$
\lambda=v(I, B)=\sup _{X \in P} \min _{i} \frac{X_{i}}{\left(B^{\mathrm{T}} X\right)_{i}}=\inf _{Y \in P} \max _{i} \frac{Y_{i}}{(B Y)_{i}} .
$$

Since $\rho(B)=\rho\left(B^{\mathrm{T}}\right)$, we find

$$
[\rho(B)]^{-1}=\sup _{X \in P} \min _{i} \frac{X_{i}}{(B X)_{i}}=\inf _{X \in P} \max _{i} \frac{X_{i}}{(B X)_{i}} .
$$

This characterization of $\rho(B)$ for $B>0$ is equivalent to Wielandt's (1950)

$$
\rho(B)=\sup _{X \in P} \min _{i} \frac{(B X)_{i}}{X_{i}}=\inf _{X \in P^{+}} \max _{i} \frac{(B X)_{i}}{X_{i}}
$$

for any irreducible $B \geq 0$. So von Neumann's theorem (4) may be seen as a step towards Wielandt's (8). 
Second, from (8), it is one more step to the formula of Birkhoff and Varga (1958) [and see Varga (1962), pp. 32, 34]

$$
\rho(A)=\sup _{X \in P^{+}} \inf _{Y \in P^{+}} \frac{X^{\mathrm{T}} A Y}{X^{\mathrm{T}} Y}=\inf _{Y \in P^{+}} \sup _{X \in P^{+}} \frac{X^{\mathrm{T}} A Y}{X^{\mathrm{T}} Y}
$$

for any irreducible nonnegative $n \times n$ matrix $A$. This formula initially suggested Corollary 2 and the strict inequality for irreducible matrices in Theorem 2, because the denominator on the right, $X^{\mathrm{T}} Y$, must be less than 1 for $X, Y \in P^{+}$. Birkhoff and Varga (1958, p. 359) observed that when $A$ is irreducible and essentially nonnegative, then the two members on the right of $(9)$ give $R(A)$, the spectral prenorm of $A$. They then stated "a curious related theorem [von Neumann's, our (1)] in game theory" and "an analogous characterization of the spectral norm" [Wielandt's, our (8)] but did not establish any connections among these minimax theorems.

Third, Donsker and Varadhan (1975) proved that if $A \geq 0$ is an $n \times n$ matrix such that $\rho(A)>0$ and $\operatorname{vec}\left[X_{i}\right]$ is the vector with $i$ th element equal to $X_{i}$, then

$$
\rho(A)=\sup _{X \in P} \inf _{Y \in P^{+}} X^{\mathrm{T}} \operatorname{vec}\left[\frac{(A Y)_{i}}{Y_{i}}\right] .
$$

See Friedland (1981, pp. 306-307) for a derivation of (10). The "other half" of (10), namely,

$$
\rho(A)=\inf _{Y \in P^{+}} \sup _{X \in P} X^{\mathrm{T}} \operatorname{vec}\left[\frac{(A Y)_{i}}{Y_{i}}\right],
$$

follows immediately from Wielandt's formula (8). Roger Nussbaum (personal communication, 29 January 1984) pointed out that (10) follows from (11) by an argument sketched by Donsker and Varadhan (1975, p. 782, bottom of column 1 and top of column 2) based on convexity and a minimax theorem of Sion (1958).

ACKNOWLEDGMENTS. We thank Lloyd Shapley for a helpful discussion and the reference to Raghavan, Roger Nussbaum for the observation above, and the referee for correcting some errors. J. E. C. is grateful for the hospitality of Mr. and Mrs. William T. Golden during this work.

Two results in our paper were discovered previously and independently. Corollary 2 is implied by a remark of Bellman $(1955$, p. 724) on the monotone convergence of an iterative procedure for computing $\rho(A)$, and Corollary 5 is stated as Theorem 1 of Raghavan (1979).

We thank T. E. S. Raghavan for bringing his paper to our attention.

\section{REFERENCES}

G. Birkhoff [1957], Extensions of Jentzsch's theorem, Trans. Amer. Math. Soc. 85, 219-227.

G. Birkhoff and R. S. Varga [1958], Reactor criticality and nonnegative matrices, J. Soc. Indust. Appl. Math. 6, 354-377.

D. Blackwell [1961], Minimax and irreducible matrices, J. Math. Anal. Appl. 8, 37-39.

M. D. Donsker and S. R. S. Varadhan [1975], On a variational formula for the principal eigenvalue for operators with maximum principle, Proc. Nat. Acad. Sci. U.S.A. 72, 780-783.

M. Dresher [1961], Games of strategy: theory and applications, Prentice-Hall, Englewood Cliffs, N.J.; reprinted as The mathematics of games of strategy: theory and applications, Dover, New York, 1981.

S. Friedland [1981], Convex spectral functions, Linear and Multilinear Algebra 9, 299-316.

F. R. Gantmacher [1960], Theory of matrices, Chelsea, New York.

L. H. Loomis [1946], On a theorem of von Neumann, Proc. Nat. Acad. Sci. U.S.A. 32, 213-215. 
T. E. S. Raghavan [1978], Completely mixed games and M-matrices, Linear Algebra Appl. 21, 35-45.

L. S. Shapley [1953], Stochastic games, Proc. Nat. Acad. Sci. U.S.A. 39, 1095-1100.

M. Sion [1958], On general minimax theorem, Pacific J. Math. 8, 171-176.

R. S. Varga [1962], Matrix iterative analysis, Prentice-Hall, Englewood Cliffs, N.J.

J. von Neumann [1928], Zur Theorie der Gesellschaftsspiele, Math. Ann. 100, 295-320; English transl., translated by Sonya Bargmann, On the theory of games of strategy, Contributions to the Theory of Games (A. W. Tucker and R. D. Luce, eds.), Vol. 4, Princeton Univ. Press, Princeton, N.J., 1959, pp. 13-42.

[1937], Ueber ein oekonomisches Gleichungsystem und eine Verallgemeinerung des Brouwerschen Fixpunktsatzes, Ergebnisse Math. Kolloq., Vol. 8, pp. 73-83.

H. Wielandt [1950], Unzerlegbare, nicht negative Matrizen, Math. Z. 52, 642-648.

R. Bellman [1955], On an iterative procedure for obtaining the Perron root of a positive matrix, Proc. Amer. Math. Soc. 6, 719-725.

T. E. S. Raghavan [1979], Some remarks on matrix games and nonnegative matrices, SIAM J. Appl. Math. 36, 83-85.

LABORATORY OF POPUlations, ROCKEFELleR UNIVERSity, NEW YORK, NEW YORK 10021

InStitute of Mathematics, Hebrew University, Givat RaM, 91904 Jerusalem, ISRAEL 